\title{
Empirical evidence suggesting a stellar upper-mass limit
}

\author{
M. Sally Oey \\ University of Michigan, Department of Astronomy, \\ 830 Dennison Building, Ann Arbor, MI 48109-1042, USA \\ email: msoey@umich.edu
}

\begin{abstract}
While theoretical understanding remains to be clarified regarding the mechanisms that may or may not limit stellar masses, it is possible to empirically evaluate the existence of an upper-mass limit. ZAMS masses of the most massive stars have been estimated for a range of environments in our local Milky Way neighborhood and the Magellanic Clouds. Various statistical techniques demonstrate the existence of an upper-mass limit in this stellar sample.
\end{abstract}

Keywords. stars: fundamental parameters, stars: mass function, stars: statistics

The stellar IMF may be treated as either a deterministic or probabilistic relation (Koen 2006). Extremely rich clusters like R136a (Oey \& Clarke 2005; Weidner \& Kroupa 2004) and the Arches cluster (Figer 2005) have enough stars that a deterministic approach can evaluate whether an upper-mass cutoff $m_{\mathrm{up}}$ exists, provided that the cluster is young enough to preclude any supernovae. Studies of these two objects find $m_{\mathrm{up}} \simeq 150 \mathrm{M}_{\odot}$. Koen (2006) uses a probabilistic approach, which assumes that the IMF is a probability density function, to evaluate the existence of a cutoff in R136a. Using distribution fitting and maximum likelihood techniques, he finds $m_{\mathrm{up}} \simeq 140-160 \mathrm{M}_{\odot}$ if the IMF slope is -2.35 ; and a steep slope of -4 to -5 if $m_{\mathrm{up}}=\infty$. Weidner \& Kroupa (2004) also emphasize that the data are consistent with no upper limit if the IMF has a much steeper slope. Ensembles of lower-mass OB associations can also be evaluated for the existence of a cutoff using a probabilistic approach. Oey \& Clarke (2005) demonstrate $m_{\text {up }}=120-150 \mathrm{M}_{\odot}$ for 9 young OB associations in the Galaxy and LMC, by compiling the distribution of probabilities of each cluster having the observed maximum stellar mass $m_{\max }$. The expected uniform distribution of these probabilities occurs only for $m_{\mathrm{up}} \simeq m_{\max }$. This illustrates results by Aban et al. (2006) who derive an estimator for the maximum value of a truncated Pareto distribution that is equal to the maximum data value. It is remarkable that these extremely different environments sampled by the Galactic center, the LMC super star cluster R136a, and ordinary OB associations, all show the same $m_{\text {up }} \simeq 150 \mathrm{M}_{\odot}$ to date. Finally, a reminder that the $\mathrm{H}$ II region luminosity function offers a probe of $m_{\mathrm{up}}$ in more distant galaxies: the existence of a flattening in its power-law slope is the manifestation of stochastic effects that are induced by an IMF having a maximum mass (Oey \& Clarke 1998).

\section{References}

Aban, I. B., Meerschaert, M. M., \& Panorska, A. K. 2006, J. Am. Stat. Assoc., 101, 270

Figer, D. 2005, Nature, 434, 192

Koen, C. 2006, MNRAS, 365, 590

Oey, M. S., \& Clarke, C. J. 1998, AJ, 115, 1543

Oey, M. S., \& Clarke, C. J. 2005, ApJ (Letters), 620, L43

Weidner, C., \& Kroupa, P. 2004, MNRAS, 348, 187 